\title{
The Monitoring and Steering Environment
}

\author{
Christian Glasner, Roland Hügl, Bernhard Reitinger, \\ Dieter Kranzlmüller, and Jens Volkert \\ GUP Linz, Johannes Kepler University Linz, \\ Altenbergerstr. 69, A-4040 Linz, Austria/Europe, \\ kranzlmueller@gup.uni-linz.ac.at, \\ http://www.gup.uni-linz.ac.at/
}

\begin{abstract}
Monitoring and steering promises interactive computing and visualization for large-scale scientific simulations. This is achieved by retrieving data about a program's execution during runtime, analyzing these data with sophisticated graphical representations, and applying changes to parameters of the simulation in progress. Based on these requirements the MoSt environment offers some novel ideas: Firstly, instrumentation of the code is performed on-the-fly during execution without a priori preparation of the target application's code. Secondly, all functionality is provided in modules which communicate with each other via a specialized network protocol, allowing the user to adapt MoSt to specific situations. Thirdly, visual representations can be generated by arbitrary visualization packages, which integrate an interface to the monitoring environment. The latter may also apply Virtual Reality techniques in order to enhance the user's understanding. This paper offers an overview of MoSt, the strategy behind it, and its main modules.
\end{abstract}

\section{Introduction}

Computational science and engineering requires not only huge amounts of processing power from high-performance computing (HPC) architectures, but also dedicated concepts, methods, and solutions incorporated in sophisticated programming and program analysis tools. Such a class of tools are computational steering environments, which offer functionality for online modifications of executing scientific and engineering simulations. In principle, there are two questions being addressed by the tools in this area:

- How to analyze a program's state at some arbitrary point during execution?

- How to modify a program (or its parameters, respectively) during execution?

Corresponding to these two questions, two distinct activities can be defined: monitoring and steering. Monitoring identifies the process of observing a program's execution during runtime, which helps the user to learn about a program's behavior. Based on the observed data, steering defines the activities applied to modify a program's execution by manipulating key characteristics of its algorithms.

V.N. Alexandrov et al. (Eds.): ICCS 2001, LNCS 2074, pp. 781790 2001.

(C) Springer-Verlag Berlin Heidelberg 2001 
Several software tools based on these two activities have been developed 15 . Some well-known examples are the on-line monitoring and steering environment FALCON [5], the Computational Steering Environment CsE [6], the Visualization and Steering Environment VASE 1], the runtime interaction framework DAQV [8] and its successor DAQV-II 9], the visualization and steering library Cumulvs [7], and the scientific programming environment ScIRUN [16].

The work described in this paper is based on some ideas derived from analyzing the tools mentioned above. Especially three aspects have been investigated:

- Attaching the monitoring part to the target application during runtime without prior instrumentation

- Using Virtual Reality technology to improve the analysis tasks, especially the problem of finding a suitable starting point for in-depth investigations

- Re-using existing visualization packages for in-depth investigations by integrating them via dedicated interfaces and efficient networking protocols.

These aspects have been implemented in the Monitoring and Steering environment MoSt. Originally intended only as a test-bed for the ideas described above, it has lately been extended to a complete environment, that it is available for usage in real-world computational science applications.

This paper is organized as follows: The next section briefly describes the main strategy behind MoST, as well as the main modules currently available. The instrumentation and monitoring module is described in Section 3. The observed data is visualized either in Virtual Reality (Section 4) or with conventional visualization tools (Section 5). Afterwards, the experiences with our MoST prototype are summarized together with an outlook on future ideas in this project.

\section{Computational Steering Strategy}

The strategy incorporated in the MoST environment can be described with a simple example. We assume, that an arbitrary scientific application is already executed on some kind of HPC system. A some time during its execution, the user decides to investigate the two questions given in Section 1, which initiates the following steps:

1. The interface of MoST is initialized on a graphical workstation.

2. With MoSt, the user logs into the HPC system and attaches the monitoring part to the target application.

3. After attaching, the user is effectively in control over the target application and starts downloading program state data.

4. The observed data is presented to the user, which in turn tries to identify critical points for in-depth investigation.

5. At these critical points, in-depth analysis is carried out, revealing more and more information about the program's execution and increasing the user's knowledge.

6. Eventually, the user identifies some key parameters, and changes their values. 


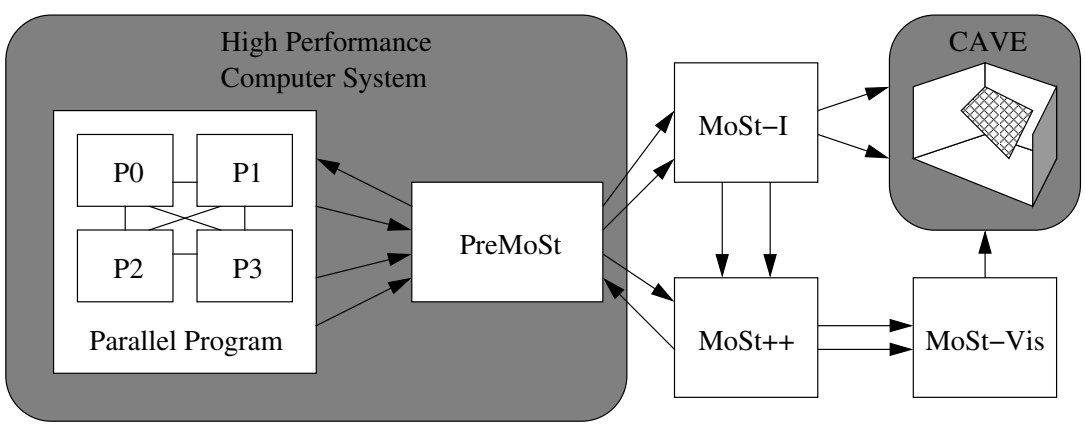

Fig. 1. Structure of the MoSt Environment

7. After the changes have been committed, MoST is detached from the target application and awaits future initiation.

This example execution of MoST demonstrates the different steps conducted during monitoring and steering. In practice, these steps are distributed among several independent modules, which communicate on a client-server basis via a dedicated networking protocol. The structure and interconnection of the MoST modules is shown in Figure 1. The following components can be identified:

- MoSt++: user interface and control center

- PreMoSt: instrumentation and monitoring module

- MoST-I: visualization of activity data in VR

- MoSt-Vis: (conventional) data visualization

The main module of MoSt is MoST ++ , which represents the control center and graphical user interface for all activities. All other modules are initiated via commands in MoST ++ , and status information as well as small amounts of analysis data (such as single scalar values) are displayed within it.

The PREMoSt module performs the instrumentation and monitoring parts of MoST. Upon initiation of MoST++, it logs into the HPC system and retrieves information about the running processes. After selecting a set of processes, PreMoSt attaches itself to the running code.

The next module initiated by MoST ++ is MoST-I, which receives activity data about the target application. This data is visualized by MoST-I within a Virtual Reality (VR) environment (e.g. a CAVE). It is used to identify critical areas or hotspots in the program, which are qualified as starting points for indepth investigation activities.

After critical areas have been detected with MoST-I, the user initiates the MoST-Vis module from the MoST++ interface. In contrast to MoST-I, which focuses on program activity data, MoST-VIS displays application dependant information, for example data structures processed by the scientific algorithms. 
The graphical representations offered by MoST-VIS are used to improve program comprehension in order to understand an application's computation. It is therefore comparable to related work in this area, which offer functionality for model exploration, algorithm experimentation, and performance optimization [15]. Additionally, the user may induce parameter changes to the running application in order to change its behavior. These changes are conducted within MoST ++ , which allows modification of program data or even code patching of a program's original functions.

Of course, the actual modifications are again carried out by PREMOST, which is actually the only module which needs to be executed on the same machine as the target application. After the monitoring and steering functions have been completed, PREMOST can be completely removed from the HPC system, leaving the original application with only the applied changes.

The next sections offer some more details about the ideas included in MoST, with special focus on the main differences between MoST and other related monitoring and steering environments.

\section{On-line Instrumentation with PreMoSt}

One of the main goals of PreMoST's design was to cope with the perturbation introduced when monitoring an arbitrary program. This is a general problem of on-line monitoring systems, because a program being observed is different from its counterpart without observation, and in some cases the observation target may even reveal completely different execution behavior 2]. Consequently, developers of monitoring tools must seek to generate as little monitor overhead as possible.

Most current approaches in this area apply dynamic monitoring [5], where the amount of overhead can be adjusted at runtime, such that less overhead is generated when less data is needed. Nevertheless, there is always a certain amount of overhead, no matter how few data is obtained. Consequently, the generated data has to be analyzed with care.

As an improvement, the approach incorporated in PREMoST does not generate any monitor overhead if no analysis data are needed. This is especially useful for long-running scientific applications, where users are not constantly monitoring the program's execution. In concrete, PrEMoST is only attached to the target application if observation is actually requested.

The on-the-fly technique included in PreMoSt is based on the dynamic program instrumentation approach described in [11, which is available in the DyninstAPI [10. Besides its main usage for performance tuning [13], DyninstAPI has also been applied to several other areas like debugging and program optimization.

DyninstAPI offers functionality to instrument an application that is already being executed on a computer system by inserting monitoring code on-the-fly. In addition, DyninstAPI can be used to establish connections between the program 
in memory and its source code by accessing the symbol table information stored in the program's object code.

Although DyninstAPI has proved very useful for our efforts, it still lacks some needed functionality. For example, access to raw memory locations are performed in DyninstAPI only via the symbol table information, which represents a certain restriction, especially for the example described in the next section. Therefore, some additional functionality had to be developed to fulfil the requirements of PreMoSt.

\section{4 "Feel the Program" with Most-I}

After PreMoSt has been attached to the program, a user needs to find out, what a target application is currently doing, or what parts of the program's data are actually being processed. The idea incorporated in MoST is to collect program activity data based on the number of accesses to a particular memory region. In concrete, the memory blocks containing the code- and data-segments of an arbitrary program are divided into areas with high activity and areas with low activity. Then, the normalized activity of an arbitrary area $A$ consisting of $n$ memory cells is computed as

$$
\operatorname{activity}(A)=\frac{\sum_{i=1}^{n} \frac{\int_{t_{\min }}^{t_{\max }} a_{i}(t) d t}{t_{\max }-t_{\min }}}{n}
$$

In this formula, the activity $a(t)$ of a memory block at a certain time $t$ is 0 , if the memory block is not accessed, or 1 , if it has been accessed recently. Furthermore, the activity over a given interval of time $\left[t_{\min }, t_{\max }\right]$ will yield higher values for memory location with more accesses.

Based on this formula, the activity of arbitrary areas or memory blocks, of distinct processes, or even a complete program can be determined. A problem is that each memory cell has to be obtained iteratively by the monitor in order to compute accurate activity values. However, as a first approximation memory blocks of coarser granularity can be selected for $a(t)$. Over time, the granularity of the cells can be refined for those blocks, that yield higher activities values in the first place. Please note, that the refinements are applied during the target program's execution, and the program's behavior is subject to change over time, Consequently, it is necessary to verify the focus of the refinement periodically. The hierarchical refinement process is described in more detail in [12.

The activity data obtained by PreMoSt is then visualized with MoSt-I. The main problem is to present the activity data of an executing program in a meaningful way to the user, so that the user understands what is happening in the program. Therefore, it was of major importance to investigate visualization techniques, which should guide the user through the activity data, where to guide means to support the user in discovering things, that were unknown before [14]. 
With the visualization, we want to improve the process of forming a mental image of the program's execution, which is utilized to aid the reasoning and understanding of program behavior [17.

This goal was addressed by Virtual Reality (VR) technology incorporated in MoST-I, where the "I" stands for Immersion. Currently, the chosen environment is the CAVE (CAVE Automatic Virtual Environment), a multi-person, roomsized, high-resolution, 3D video and audio environment developed in 1992 at EVL (Electronic Visualization Laboratory, University of Illinois at Chicago). The task of MoST-I is to generate the immersive model of the program's behavior in the CAVE. Then the user may interactively explore this virtual world and gain more and more knowledge about the program's characteristics.

The idea of using VR in MoST-I is, that it allows to cause a "feeling" in the user about the program's behavior. By exploring the activity data presented in the CAVE, the user can intuitively investigate even huge amounts of data. This can mainly be attributed to the fact, that VR offers additional dimensions compared to traditional 2-dimensional displays. Some important characteristics in this context are: Firstly, the number of available dimensions is increased (e.g. 3D, immersion, sound). Secondly, important issues can be emphasized by combining attributes (e.g. color+sound). Thirdly, animation can be used to describe the variation of data over time.

A major difficulty for visualization is always to choose an appropriate visual representation. For MoST-I several approaches have been investigated. The most suitable of these approaches seems to be the activity tunnel, which arranges the memory of parallel processes as stripes in a 3-dimensional tunnel [12]. Within this tunnel, the activity is visualized with attributes like color, shades, object size, and animation.

An example of the activity tunnel is presented in Figure2. It shows the memory activity of 8 application processes, with different shades indicating different levels of activity. This picture has been taken within the CAVE environment and also contains the user who controls the application. In order to intensify the effect of VR onto the user, all interaction with the system is performed via voice input, which is shown in Figure 2 with the mounted headset.

In addition to the graphical representation, mappings to other sensory input are currently investigated. One example is sound, which was originally inspired by the following story about the Whirlwind computer (1950) [4]: You even had audio output in the sense that you could hear the program because there was an audio amplifier on one of the bits of one of registers - so each program had a signature. You could hear the tempo of how your program was running. You could sense when it was running well, or when it was doing something surprising.

Similar ideas are discussed in [3. By integrating sound output in MoST-I, we try to emphasize the activity of a program in the region of the tunnel, that is currently inspected by the user. Other ideas include the sonification of hardware performance counters, the program counter, and similar state data. 


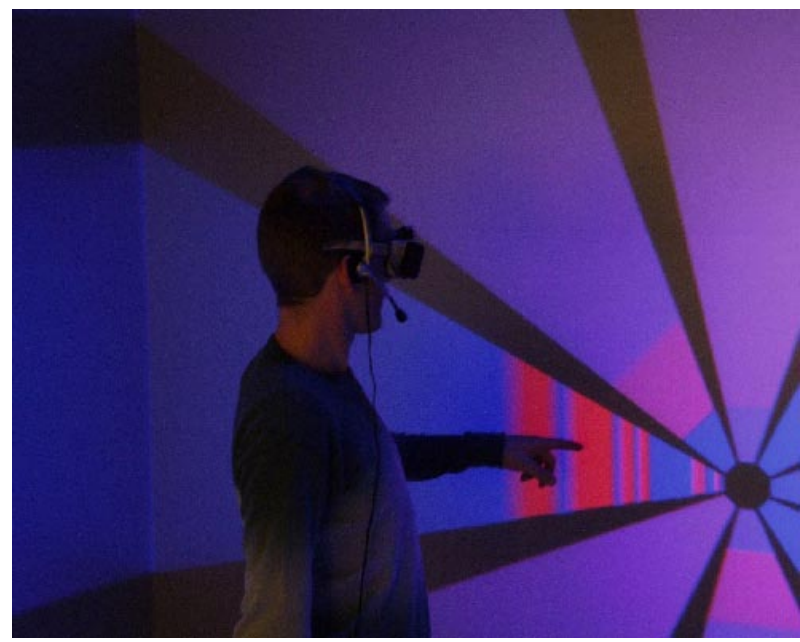

Fig. 2. Picture of user (with microphone) and the activity tunnel in the CAVE.

\section{Data Visualization with MoSt-Vis}

As described above, the visualization MoST-I is mainly used as a starting point for in-depth investigations. Therefore, after the user has decided where to perform program and data analysis, symbol table information is presented by MoST ++ . Based on this information, interesting data structures of the running program can be selected. The selected data is then extracted by PreMoSt. Depending on the amount of data, the results of PrEMoST are either printed in a window of $\mathrm{MoST}_{+}+$, or forwarded to the visualization module MoST-VIS.

The graphical representations provided by MoST-VIS have to be chosen corresponding the the scientific applications data. Since most of these data is already visualized during post-mortem analysis, it seems beneficial to re-use existing visualization packages. This is achieved within MoST by developing corresponding input interfaces, such that PREMOST can forward its data to an arbitrary visualization tool. At present, input interfaces for IBM's Open Data Explorer (OpenDX) are available, while similar functions are currently being implemented for AVS/Express.

Some examples of data visualization are shown in Figure 3 to Figure [5, All screenshots have been produced with MOST while the target application was actually running. Figure 3 shows the scalar value of a chosen variable over time, with each curve corresponding to one process of the application. Figure 4 shows a two-dimensional heat-diagram of an array, that is currently processed by the target application. Figure 5 shows a 3 -dimensional visualization of a crank shaft grid, that is currently used for computing the stress around this object.

The visualizations are constructed within MoST as follows: MoST++ reads the symbol table information and presents the set of available data structures 


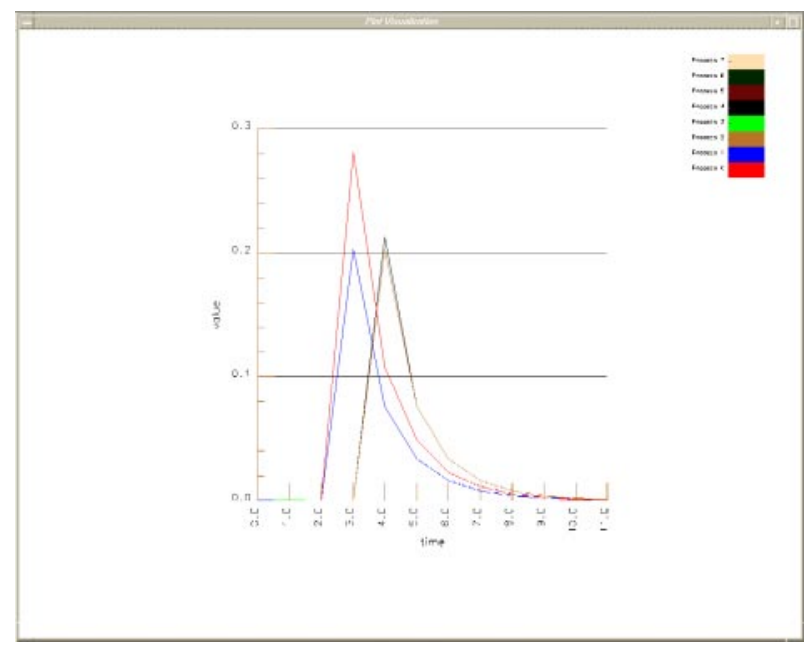

Fig. 3. Example visualization of scalar values on 8 processes over time

to the user. Upon choosing some of the data structures, PreMoSt begins to download the corresponding data from the running program. In addition, the user selects a suitable graphical representation within $\mathrm{MoST}++$ and specifies the mapping of the data structures onto the dimensions of the graphical output. These data and the mapping specification are forwarded to MoST-VIS which finally generates the output described above.

\section{Conclusions}

The MoSt environment described in this paper offers some novel ideas for analyzing and controlling applications from computational science and engineering. Although originally intended only to verify some of our ideas, the first results with the tool where rather promising. This convinced us to develop the system into an environment, that can be offered interested scientists.

Most useful is the dynamic instrumentation module, which offers to inject commands on-the-fly. Therefore the application needs not be stopped and restarted, which allows to decrease the overall computation time, a factor that may be crucial for users of expensive HPC systems.

On contrary, the VR visualization of activity data may seem a little bit controversial in the first place. However, the problem of leading the user to an suitable starting place for in-depth investigations is very important, and there seems to be only limited support from related work in this area. Nevertheless, it is certainly necessary to assess the usefulness of the current approach for real-world applications, and to continue studying this important aspect.

Finally, the idea of re-using existing visualization tools by developing corresponding interfaces allowed to shift lots of concentration to the other aspects 


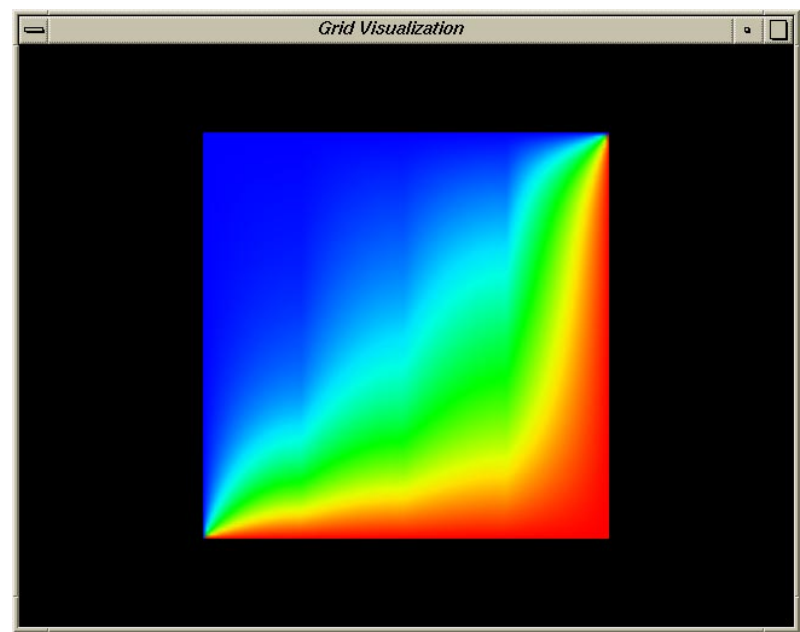

Fig. 4. Example heat diagram of 2-dimensional data array

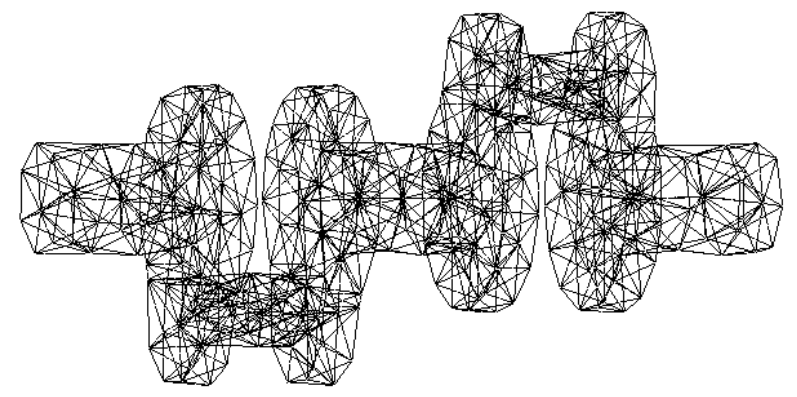

Fig. 5. Example 3-dimensional data visualization of a crank shaft

of this project. The problem in this area is mainly related to the efficiency of the networking protocol and the underlying network infrastructure. Especially for tools with huge amounts of data, dedicated methods for filtering or preprocessing need to be developed.

\section{References}

1. Brunner, J.D., Jablonowski, D.J., Bliss, B., Haber, R.B., "VASE: The Visualization and Application Steering Environment", Proc. Supercomputing '93, Portland, OR, pp. 560-569 (November 1993).

2. Eisenhauer, G., Gu, W., Kraemer, E., Schwan, E., Stasko, J., "Online Displays of Parallel Programs: Problems and Solutions", Proc. PDPTA '97, Intl. Conference on Parallel and Distributed Processing Techniques and Applications, Las Vegas, NV, pp.11-20 (July 1997). 
3. Francioni, J.M., Jackson, J.A., "Breaking the Silence: Auralization of Parallel Program Behavior", Journal of Parallel and Distributed Computing, Vol. 18, pp. 181194 (1993)

4. Frenkel, K.A., "An Interview with Fernando Jose Corbató", Communications of the ACM, Vol. 34, No. 9, pp. 83-90 (September 1991).

5. Gu, W., Eisenhauer, G., Schwan, K., Vetter, J., "Falcon: On-line Monitoring and Steering of Large-Scale Parallel Programs", Concurrency: Practice and Experience, Vol. 10, No. 9, pp. 699-736 (August 1998).

6. van Liere, R., Mulder, J.D., van Wijk, J.J., "Computational Steering", Future Generation Computer Systems, Vol. 12, No. 5, pp. 441-450 (April 1997).

7. Geist, G.A., Kohl, J.A., Papadopoulos, P.M., "CUMULVS: Providing FaultTolerance, Visualization, and Steering of Parallel Applications", Intl. Journal of Supercomputer Applications and High Performance Computing, Vol. 11, No. 3, pp. 224-236 (Fall 1997).

8. Harrop, Ch.W., Hackstadt, S.T., Cuny, J.E., Malony, A.D., Magde, L.S., "Supporting Runtime Tool Interaction for Parallel Simulations", Proc. Supercomputing '98, Orlando, FL (November 1998).

9. Hackstadt, S.T., Harrop, Ch.W., Malony, A.D., "A Framework for Interacting with Distributed Programs and Data", Proc. HPDC-7, 7th IEEE Intl. Symposium on High Performance Distributed Computing, Chicago, IL, pp. 206-214 (July 1998).

10. Hollingsworth, J.K., Buck, B., "DyninstAPI Programmer's Guide - Release 2.0", Computer Science Department, URL: http://www.cs.umd.edu/projects/dyninstAPI (2000).

11. Hollingsworth, J.K., Miller, B.P., Cargille, J., "Dynamic Program Instrumentation for Scalable Performance Tools", Proc. 1994 Scalable High Performance Comp. Conf., Knoxville, TN (1994).

12. Kranzlmüller, D., Reitinger, B., Volkert, J., "Experiencing a Program's Execution in the CAVE", Proc. PDCS 2000, Conference on Parallel and Distributed Computing Systems, Las Vegas, NV, USA, pp. 259-265 (November 2000).

13. Miller, B.P., Callaghan, M.D., Cargille, J.M., Hollingsworth, J.K., Irvin, R.B., Karavanic, K.L., Kunchithapadam, K., Newhall, T., "The Paradyn Parallel Performance Measurement Tool", IEEE Computer, Vol. 28, No. 11, pp. 37-46 (November 1995).

14. Miller, B.P., "What to Draw? When to Draw? - An Essay on Parallel Program Visualization", Journal of Parallel and Distributed Computing, Vol. 18, No. 2, pp. 265-269 (June 1993).

15. Mulder, J.D., van Liere, R., van Wijk, J.J., "A Survey of Computational Steering Environments", Future Generation Computer Systems, Vol. 15, No. 1, pp. 119-129 (February 1999).

16. Parker, S.G., Johnson, C.R., "SCIRun: A Scientific Programming Environment for Computational Steering", Proc. Supercomputing '95, San Diego, CA, (December 1995).

17. Zhang, K., Ma, X., Hintz, T., "The Role of Graphics in Parallel Program Development", Journal of Visual Languages and Computing, Academic Press, Vol.10, No.3, pp. 215-243 (June 1999). 